\title{
Micro-usinage par impulsions laser ultra-courtes
}

\author{
E. Baubeau, R. Le Harzic, E. Audouard, C. Jonin, M. Courbon, S. Mottin \\ et P. Laporte
}

\section{Laboratoire Traitement du Signal et Instrumentation, Université Jean Monnet, UMR 5516 du CNRS, 23 rue du Docteur Paul Michelon, 42023 Saint-Etienne cedex 2, France}

\begin{abstract}
Résumé: Ce travail présente des résultats de micro-usinage femtoseconde, utilisant une chaine Tisaphir à $1 \mathrm{KHz}$. Le cas des métaux est particulièrement envisagé et les taux d'ablation par tir est déterminé dans le cas du cuivre ( $48 \mathrm{~nm} / \mathrm{tir})$ et de l'inox ( $29 \mathrm{~nm} / \mathrm{tir})$.
\end{abstract}

\section{Introduction}

L'utilisation d'impulsions ultra-courtes (de l'ordre de quelques dizaines de femtosecondes) ouvre de nombreuses perspectives à la fois industrielles et scientifiques. En particulier, pour le micro usinage, il est possible d'atteindre en mode femtoseconde le seuil d'ablation de tous les types de matériaux. On peut citer les métaux spéciaux et céramiques utilisés en milieu médical, les céramiques piézo-électriques, le silicium et les matériaux organiques pour la micro-électronique par exemple, ainsi que les matériaux pour la micro-mécanique, etc. L'usinage est alors très précis (de l'ordre du micron et même inférieur), sans bavures et avec une ZAT (Zone Affectée Thermiquement) très réduite.

\section{Dispositif expérimental}

Pour valider les processus de micro-ablation, nous avons utilisé une source femtoseconde Tisaphir de cadence $1 \mathrm{KHz}$ ( $0.5 \mathrm{~mJ} / \mathrm{impulsion}, 170 \mathrm{fs}, 800 \mathrm{~nm}$, Pmoy $0.5 \mathrm{~W}$ ). Le faisceau est filtré spatialement par l'introduction d'un diaphragme de diamètre $5 \mathrm{~mm}$. Le micro-usinage est réalisé soit au point focal, soit dans un plan image du diaphragme. Le faisceau est fixe et l'échantillon est piloté par des platines de translation ( 3 axes) précises au micron, la vitesse maximale de déplacement étant de $20 \mathrm{~mm} / \mathrm{s}$. Le faisceau est focalisé sur l'échantillon par une lentille de $50 \mathrm{~mm}$ de focale.

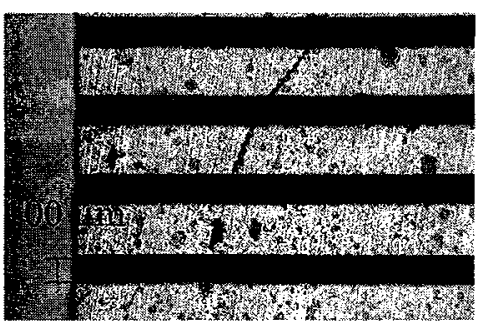

Fig 1 : Micro-usinage dans un échantillon de cuivre sous incidence normale et à vitesse constante de $1 \mathrm{~mm} / \mathrm{s}$, pour $2,4,6,8$ passages du faisceau laser ( $\mathrm{du}$ bas vers le haut)

\section{Résultats et discussion}

La figure 2 a) présente les résultats d'expériences de micro-usinage de métaux d'une part pour du cuivre (famille des métaux nobles) et d'autre part pour de l'inox (alliage, se rapprochant des métaux de transition). La profondeur d'ablation est représentée en fonction du nombre de passages de l'échantillon devant le faisceau laser pour différentes vitesses de translation. On remarque que cette profondeur d'ablation est linéaire en fonction du nombre de passages. De plus l'absence d'effet thermique permet de conserver la même qualité d'usinage quel que soit 
le nombre de passages, ce qui donne donc la possibilité de contrôler la profondeur d'usinage. Une des raisons pouvant expliquer la différence d'efficacité pour les mêmes conditions expérimentales dans le cas du cuivre et de l'inox est la différence de comportement électronique du fait du couplage électron-phonon [1,2]. Ce couplage étant plus important pour l'inox que pour le cuivre, les électrons thermiques diffusent moins dans l'inox que dans le cuivre, la zone adressée par le rayonnement puis ablatée est donc plus grande dans le cuivre que dans l'inox.

De plus, comme la figure 2 b) l'illustre, la profondeur d'ablation étant inversement proportionnelle à la vitesse de déplacement des platines, on déduit des mesures le taux d'ablation par tir. Dans nos conditions expérimentales, on trouve un taux d'ablation pour le cuivre de $48 \mathrm{~nm} / \mathrm{tir}$, et pour les mêmes conditions de $29 \mathrm{~nm} /$ tir pour l'inox.

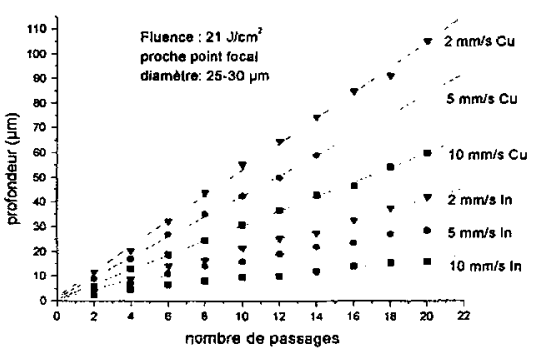

Fig 2 a) : Profondeur de matière ablatée en fonction du nombre de passages pour le Cuivre et l'Inox

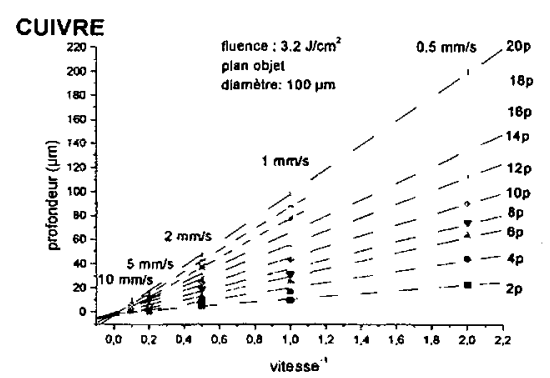

Fig 2 b) : Profondeur de matière ablatée en fonction de la vitesse pour le cuivre, pour $x$ passages

\section{Perspectives}

Les développements futurs utiliseront une chaîne femtoseconde pouvant atteindre 15 à 20 $\mathrm{KHz}(0.5 \mathrm{~mJ} / \mathrm{impulsion}, 50 \mathrm{fs}$, Pmoy :10W). L'originalité de cette chaîne repose sur le recours à un laser de pompe $\mathrm{CuHBr}(70 \mathrm{~ns}, 511$ et $578 \mathrm{~nm}, 5$ à $25 \mathrm{KHz}$, Pmoy :100 W) développé au Laboratoire de Spectrométrie Physique de Grenoble [3].

Ce prototype s'insérera dans une plate-forme laser femtoseconde à vocation recherche et applications industrielles, développée à St Etienne par le laboratoire TSI en collaboration avec le Pôle Optique et Vision et MECAPOLE, et qui proposera une gamme encore plus large de sources femtosecondes orientées vers l'étude de procédés et le travail de petite série.

\section{Références}

1. P.B. Corkum, F. Brunel and N.K. Sherman, Phys. Rev. Lett. 61, 2886 (1988).

2. S.-S. Wellershoff, J. Hohlfeld, J. Gudde, E. Matthias, Appl. Phys. A 69 (1999).

3. P. Coutance, G. Naylor and J.-P. Pique, IEEE J. Quantum Electron., 31, 10 (1995). 